\title{
La psicología penitenciaria: modos de comprender la intervención psicológica por parte de los internos.
}

\author{
Penitentiary psychology: ways of understanding psychological \\ intervention from inmates' perspective.
}

Esta obra está bajo una Licencia Creative Commons Atribución 4.0 Internacional. DOI: $\underline{10.32870 / \text { sincronia.axxiii.n75.19a19 }}$

Luis Jaime González Gil

Instituto Tecnológico y de Estudios Superiores de Occidente

luisj.gonzalez.gil@gmail.com

(MÉXICO)

Roxana Adib Jonsson.

Instituto Tecnológico y de Estudios Superiores de Occidente

Ana Belén Leal Lliteras.

Instituto Tecnológico y de Estudios Superiores de Occidente

Natalia Hernández Michel Rizo.

Instituto Tecnológico y de Estudios Superiores de Occidente

Paola Salas Romo.

Instituto Tecnológico y de Estudios Superiores de Occidente

Recibido: 29/10/2018

Revisado: $16 / 11 / 2018$

Aprobado: 04/12/2018

\section{RESUMEN}

La psicología es uno de los principales saberes que participan en la legitimación y construcción de la realidad penitenciaria como centros de readaptación, y no tanto como lugares de encierro o calabozos. Bajo el trabajo de los psicólogos, los internos no sólo son castigados y privados de su 
libertad sino también intervenidos desde distintas corrientes clínicas, sociales y comunitarias en pro de su reinserción social. Por ello, la presente investigación, a partir de una metodología cualitativa de corte etnográfica, le dio un giro a la discusión, analizando la forma en que los internos privados de libertad (que consumen el tratamiento) comprenden la figura del psicólogo y viven su tratamiento psicológico. Los resultados muestran que la psicología se comprende desde la dicotomía aprobación/desaprobación a partir de su institucionalización latente o no latente. La desaprobación se genera cuando se le interpreta como una extensión de la institución en donde el psicólogo funge como un espía encubierto y la psicología como un saber estigmatizante.

Palabras clave: Psicología. Reinserción. Prisiones. Poder.

\section{ABSTRACT}

Psychology is one of the main knowledges that participate in the legitimization and construction of penitentiary reality as rehabilitation centers and not as places of confinement or dungeons. Under the psychologists' work, inmates are not only punished and deprived of their freedom but also intervened following different clinical, social and community currents in favor of their social integration. Therefore, this research, based on a qualitative ethnographic methodology, gives a different perspective to the discussion, analyzing the ways in which the inmates deprived of freedom experience the psychological treatments and understand the psychologist role and figure. The results show that psychology is understood from an approval/disapproval dichotomy starting with its latent or not latent institutionalization. The disapproval is generated when it is interpreted as an extension from the institution, where the psychologist functions as an undercover spy and psychology as a stigmatizing knowledge.

Keywords: Psychology. Social Reintegration. Prisons. Power.

\section{Introducción}

El contexto penal mexicano, a grandes rasgos, presenta ciertas paradojas que se originan en la misma institución y en la división dicotómica de quienes, por un lado, hacen las leyes 
institucionales, y por otro, aquellos a quienes van destinadas. En términos concretos, se ha olvidado que el tratamiento del penado, para lograr la presunta reinserción social, debería de tomar en consideración la posición del sujeto al que se le privará de su libertad, en lugar de pensar únicamente en un sistema de adoctrinamiento disciplinario (Foucault, 1975) generado por un modelo penal construido por aquellos que no reconocen la experiencia de vivir en el encierro, 0 aquellos que están fuera de la institución, o incluso, que nunca han estado en ella.

Esto se hace aún más evidente en términos estructurales. Como menciona Sanz (2004), cuando argumenta que incluir excluyendo es una contradicción latente. Además como menciona Bergalli (1976) "resulta eufemístico un régimen que proclama la autodisciplina con fines readaptadores y luego impone autoritariamente normas de conducta (pp. 35)”. El hecho de que la prisión, además de separar al delincuente de la sociedad (convirtiéndolo en preso), también busca, o al menos lo aparenta, reinsertarlo en la sociedad, aspirando a un ideal de persona rehabilitada, transformada y arrepentida de sus actos delictivos; resulta sumamente paradójico.

Asimismo, el problema está inserto en las condiciones de la institución: en la convivencia carcelaria se vive con reglas y hábitos contradictorios al prototipo del "buen ciudadano", esto debido al impacto desadaptador que se vive dentro de la cárcel. Según García-Borés (2003) este efecto en las personas privadas de libertad depende de múltiples factores de distinta índole: 1) social porque influye su manera de vincularse con el exterior, sus condiciones sociales, laborales y económicas; 2) personal, en cuanto a características de personalidad, edad, contexto familiar; y 3) las propias circunstancias penales-penitenciarias, como el tiempo de condena. En consecuencia, éstas condiciones producen que el sujeto tenga una difícil adaptación fuera del encierro ya que, por sí mismas, se insertan dentro de una lógica de desadaptación, al propiciar que el sujeto pierda u olvide las facultades vitales y sociales necesarias para llevar a una vida en libertad (Sanz, 2004).

Ahora bien, independientemente de este tipo de incongruencias, actualmente la reinserción se intenta lograr por medio de distintos saberes. Uno de ellos es(son) el(los) discurso(s) psicológico(s) que actúa como una ciencia especializada en producir un cambio en el sujeto 
mediante un trabajo terapéutico, legitimando ciertos comportamientos y valores que deben ser adoptados por los internos para reinsertarse con éxito.

En términos académicos, esta discusión se ha extendido a dos dimensiones: la naturaleza y causa del delito y la búsqueda de las mejores formas de intervenir en la institución. En cuanto al primero, Baratta (1986) y Pavarini (1983) muestran cómo existen un conjunto de saberes hegemónicos que delimitan al sujeto delictivo desde un enfoque positivista y determinista del actuar humano. El problema se encuentra en el individuo, y como consecuencia, la idea de la reinserción social se dirige a la identificación del problema (especie de diagnóstico) desde disciplinas como la psicología, el psicoanálisis, la criminología, el trabajo social o la sociología.

En cuanto al segundo, que se desprende del primero, el debate se centra en establecer qué tipo de tratamiento es efectivo para producir un cambio en el sujeto. Se proponen, así, programas de desarrollo y fortalecimiento de los aspectos carenciales de los internos (Herrero y León, 2006), talleres de Arteterapia y expresión (Conde y Tejedor, 2009), talleres de escritura y expresión (Heleusa, 2006), trabajo como dispositivo de educación en las cárceles (R. De Alós, Artiles, A, Migueléz, F \& Gibert, F., 2009), trabajo comunitario (Sanz, 2004), o planes críticos que plantean hacer menos cárcel la cárcel (Kouyoumdjian y Poblet, 2010), entre otros.

Sin embargo, siguiendo a Foucault (1975) pareciera que más que contribuir a detectar el problema y solucionarlo con un programa, la psicología se ha traducido en un dispositivo de poder y legitimización del discurso penal-jurídico. Como efecto colateral, se construye un lenguaje terapéutico que patologiza y margina al interno, constituyéndolo conceptualmente como un sujeto desviado, anormal, con falta de habilidades sociales, en contexto de alto riesgo, o sin educación. Parafraseando a García-Bores (1995) se construye la figura del delincuente desde el "paradigma etiológico de la criminalidad", el cual, define al individuo particularmente afectado por unas u otras razones pero que, en definitiva, es el contenedor del conflicto; sea biológica, social o psicológicamente la raíz del problema se encuentra en o alrededor del interno. 
La psicología, entonces, está también inmersa en las incongruencias enunciadas anteriormente, ya que es utilizada para fines de custodia y orden, enfocándose por igual en la visión institucional. Los discursos psicológicos, al explicar la realidad desde su enfoque, valida los métodos utilizados para la reinserción, envolviendo a todas las personas que han cometido un delito en las mismas condiciones de justicia criminal: el reclutamiento en la cárcel para el tratamiento individual.

En este sentido, bajo estas condiciones estructurales, se manifiesta la pertinencia de investigar la voz de los que consumen los programas de reinserción de carácter psicológico (terapia y talleres). Esto con el objetivo de visibilizar cómo las personas (internos) que son el objetivo de los programas de reinserción comprenden y experimentan la psicología (y a los psicólogos) desde su experiencia penitenciaria, inserta en estas incongruencias; cuestión que posibilitaría ir más allá del enfoque institucional que no incluye la perspectiva de los internos por motivos de control y vigilancia.

\section{Marco Teórico}

Foucault (1975) plantea que el poder se encuentra en todos lados, difundido e incorporado en discursos, saberes y regímenes de verdad que producen formaciones sociales, así como realidades. Se ejerce por medio de un entretejido de relaciones entre dominadores y dominados, reflejo de la estructura del cuerpo social actual, dentro y fuera del centro penitenciario. Este poder consta de ciertos mecanismos para ejercerse, como lo son el castigo, la disciplina y la vigilancia; mismos que permiten la producción, acumulación y funcionamiento de saberes que normalizan. Para el autor, existe una relación saber-poder: se produce un saber del interno, desde el cual, puede ejercerse poder y dominio sobre él. Al mismo tiempo, el sujeto puede apropiarse e interiorizar ese conocimiento y utilizarlo.

Foucault (1999) entiende el discurso como prácticas sociales que producen realidades, y son hechas visibles por las palabras y las acciones; no son un simple soporte en que se manifiesta el poder; por el contrario, el discurso es aquello por lo que, y por medio de lo cual se puede ejercer el 
poder. Las prácticas discursivas, incluidas las psicológicas, se consideran como reglas anónimas, construidas en el proceso histórico, es decir, determinadas en el tiempo y espacio y, de esta manera, se van definiendo las condiciones que hacen posible cualquier enunciación.

Desde la teoría del construccionismo social, Gergen $(1996,1997)$, afirma que los significados que se tienen de la realidad no están dados por sí solos, sino que dependen de los grupos sociales que los construyen. La veracidad de cualquier enunciado sobre la realidad sólo es determinada por el nivel de argumentación y la posición en la red conversacional que tenga quien lo enuncia, por tanto, es un consenso derivado de una interacción social.

Según el enfoque que plantea este autor, las concepciones sobre la identidad se derivan del proceso social, considerándola como un discurso acerca del yo, como una narración producto del intercambio social. Además, las exposiciones narrativas que están incrustadas en la acción colectiva, hacen que los acontecimientos sean visibles y establecen expectativas para situaciones futuras. Dado que los sucesos de la vida cotidiana están inmersos en la narración, se van cargando de sentido relatado. La manera en que las personas se significan a sí mismas, a los otros y a la realidad, depende del intercambio social y de las formas narrativas.

Las significaciones construidas colectivamente dan pie a que se validen y normalicen las relaciones de poder y el funcionamiento institucional dentro de un centro penitenciario. Pero al introducirnos en esta red relacional entre internos y personal institucional, se pueden encontrar resquicios en donde no siempre la institución funciona de manera totalizadora (Goffman, 2001).

Por su parte, De Certeau (1980), explica la estrategia, táctica y consumo para dar cuenta de la posibilidad de que el ejercicio del poder sea subvertido y alterado en su significado por las prácticas cotidianas. Este autor explica la estrategia como las acciones que pueden planificarse previamente, ésta una estructura que se piensa sobre el espacio y el dominio de los lugares mediante la vista. Refiriéndose a la expresión del poder mediante un espacio que se reconoce como propio, donde se delimitan normas y objetivos; se prevén situaciones y se analizan las posibles amenazas, para así ejercer el poder. 
Al contrario de la estrategia, la táctica se aprovecha de las fallas, de las ocasiones e intenta filtrarse y moverse dentro de las estrategias. Para operar tácticamente se necesita actuar de manera imprevista y transformar una situación en un beneficio. No se planea, ni se piensa previamente de manera estructural, la táctica es espontánea, aparece en el momento y trata de enfrentarse a la situación.

Por su parte, Goffman (2001) describe que dentro de las instituciones existen los llamados ajustes secundarios, éstos son prácticas que, sin desafiar directamente al personal institucional, permiten a los internos obtener placeres prohibidos, o no prohibidos, mediante medios no lícitos. Esta necesidad de obtener satisfacciones personales de los internos, se da justamente, como plantea el autor, al momento de ser privados de su libertad, pues los internos entran a un proceso en el que se les impone poco a poco un papel que los desidentifica de su anterior yo. Lo descrito provoca una mortificación del yo, ya que su identidad anterior es nulificada, atacada y estigmatizada por la sociedad, por lo que se busca reformar a la persona a una identidad socialmente aceptada.

Todo lo anterior da pie a pensar el espacio social, en este caso el centro penitenciario, como el resultado de relaciones de poder y la resistencia ante estas; un producto del intercambio social y de las significaciones que lo orientan, lo sitúan y permiten su funcionalidad. Las estrategias de reinserción empleadas por la institución son un reflejo de discursos, como los psicológicos, desde los cuales opera la cárcel. No obstante, así como la parte dominante, o sea la institución, se posiciona desde ciertos saberes y construye una manera normalizada de entender y tratar a los internos; ellos también construyen significados acerca de los discursos psicológicos, así como de sí mismos y de su estar en la cárcel.

\section{Marco metodológico}

La presente investigación se realizó utilizando la metodología cualitativa, la cual pretende comprender un fenómeno desde el interior, mediante una indagación profunda, elaborada 
primordialmente en el escenario de los sujetos. La metodología cualitativa facilita la comprensión de múltiples realidades, tomando en cuenta que estas se construyen por los diferentes actores y las interacciones entre ellos. Entonces, se aparta de la idea de encontrar una realidad objetiva ya que el conocimiento es una producción constructiva e interpretativa. (González, 2002). Con base en este paradigma, se diseñó un taller que fue utilizado como la principal técnica implementada para recabar datos. Este se realizó desde un paradigma con corte etnográfico (mediante la observación participante) y basado en la Pedagogía del Encuentro (Godenzi, 1999), el cual, fija su atención en la dimensión pragmática del lenguaje que es el diálogo, y su principal herramienta, la conversación.

El taller se llevó a cabo dentro de las instalaciones de un centro penitenciario juvenil del estado de Jalisco. La población constó de aproximadamente entre 15 y 23 jóvenes privados de libertad, de 18 a 21 años de edad. Específicamente, el taller fue dirigido por cuatro estudiantes de la licenciatura en psicología, apoyadas y asesoradas por el coordinador del proyecto. Durante los talleres se asignaron roles para la distribución del trabajo: un coordinador, un facilitador y dos participantes. El coordinador dirigía la actividad pensada para el día, fungiendo como guía, encargado de explicar y dar las indicaciones. El facilitador se encargaba de tener todos los elementos necesarios para la realización de la actividad, como la duración de cada una de ellas, el material y además actuaba como el principal soporte del coordinador. Mientras que las otras dos talleristas se incorporaban a la actividad, asistiendo a los internos, al facilitador y coordinador. A pesar de los roles asignados, las funciones de cada tallerista eran adaptables a las situaciones presentadas en las sesiones.

El taller se realizó con el objetivo general de crear un espacio para la expresión e intercambio de experiencias dentro y fuera del espacio penitenciario. Se definieron como objetivos específicos: identificar la manera en que los internos perciben y resuelven los diferentes conflictos planteados en las actividades; las figuras de autoridad y los discursos que éstas promueven; así como conocer las diferentes perspectivas que tienen los internos acerca de su estar y devenir penal. Posteriormente, a partir del desarrollo de los talleres y mediante las interacciones con los internos, 
se definió el objetivo de investigación, siendo este: conocer la manera en que los internos significan los discursos psicológicos (Ver Tabla 1)

Tabla 1. Comparación de los talleres en el periodo uno y dos.

\begin{tabular}{|c|c|c|}
\hline & Primer periodo & Segundo Periodo \\
\hline Tema/título & "Taller de encuentro" & "Género" \\
\hline Periodo & $\begin{array}{l}\text { Octubre } 2016 \text { - Diciembre } \\
2016\end{array}$ & Febrero 2017 - Abril 2017 \\
\hline Sesiones & 8 sesiones & 10 sesiones \\
\hline Horario & Martes 10:30 am - 12:00 p.m & Martes 2:00 pm - 3:30 pm \\
\hline Asistentes & $\begin{array}{l}\text { Módulo no. } 3 \text { y } 5 \text { (aprox. } 22 \\
\text { internos, dependiendo de la } \\
\text { asistencia) }\end{array}$ & $\begin{array}{l}\text { Módulo no. } 5 \text { (aprox. } 16 \\
\text { internos, dependiendo de la } \\
\text { asistencia) }\end{array}$ \\
\hline $\begin{array}{l}\text { Objetivo } \\
\text { General }\end{array}$ & $\begin{array}{l}\text { Identificar la concepción que } \\
\text { tienen los internos de los } \\
\text { roles de género, y entender } \\
\text { cómo desempeñan los } \\
\text { propios. }\end{array}$ & $\begin{array}{l}\text { Generar un espacio de } \\
\text { encuentro que propicie la } \\
\text { expresión e intercambio de las } \\
\text { experiencias de los internos } \\
\text { dentro de la institución } \\
\text { penitenciaria. }\end{array}$ \\
\hline
\end{tabular}

Feunte: Elaboración propia

Se planearon dos actividades por sesión de taller, con aproximadamente 45 minutos de duración cada actividad y 15 minutos de receso entre ambas. Las actividades estaban pre-diseñadas mediante un cronograma, pensado principalmente con modalidad lúdica e interactiva, para así 
fomentar un espacio dinámico, de socialización, interacción y diálogo, sin dejar a un lado los objetivos del taller y de la investigación. A lo largo del taller, se realizaron modificaciones que dependían de cómo se desarrollaba cada sesión, haciendo los cambios necesarios para un mejor desarrollo del mismo.

La observación participante permitió a las talleristas construir un taller pensado de manera activa, tomando en cuenta la participación de estos en las actividades, quienes también formaban parte de la interacción dentro del escenario. Por lo tanto, el taller cumplía dos funciones: el desarrollo de la temática propuesta, y a la vez, el objetivo de la investigación. Además, al mismo tiempo, se contempló la relación talleristas-internos mediante el diálogo y entrevistas no estructuradas y/o semi-estructuradas, que abonaban al tema de la investigación.

Asimismo, se realizaron bitácoras de cada sesión impartida, las cuales constaron en la narración de las interacciones de cada sesión, incluyendo los hechos, las interpretaciones y la unión con la teoría. En el primer periodo de talleres, las bitácoras eran escritas de manera personal, es decir, se obtuvieron 7 bitácoras por tallerista. Sin embargo, en el segundo periodo, se realizaron en equipo las 9 bitácoras, una por sesión, sumando así un total de 37 bitácoras. Esto permitía la discusión de lo que acontecía dentro de la institución, ya que no era permitido entrar con grabadoras o cualquier tecnología que materializara el momento.

Por último, el análisis de los datos se realizó a partir de la técnica denominada análisis temático, la cual, permitió proporcionar patrones o temas a partir de una cuidadosa lectura y relectura de la información recogida (Braun y Clarke, 2006). Se trató de un proceso en el que los objetivos de investigación funcionaron como palancas para identificar unidades de registro que estuvieran relacionadas a estos. No obstante, en el proceso de análisis, fueron re-leídas y reorganizadas las unidades de registro para otorgarles un sentido temático de manera inductiva, y no tanto deductiva como en el primer proceso (utilización de los objetivos para identificar y organizar la información). 


\section{Resultados}

\section{-La psicología en y por las tensiones de poder}

La psicología, desde la forma en que la entienden los internos, se instituye, a mayor y menor grado, a partir de la relación que mantiene con el poder institucional. En primer lugar, porque se establece desde un discurso que dicta lo que es normal y anormal, es decir, que opera desde una lógica mortificante y estigmatizante desde la categoría loco, produciendo rechazo en el interno). En segundo lugar, la psicología se encuentra inserta en un mecanismo de control que está al servicio del aparato jurídico: la labor del psicólogo no sólo interviene en la subjetividad del interno, sino, además, tiene repercusión en la decisión de los jueces o personas encargadas de llevar el caso.

De este modo, en general, la psicología se percibe como un aparato que genera un saber del interno e influye en el dominio del espacio penitenciario, así como en la producción de reglas y limitaciones. No obstante, se produce, en contraparte, un juego de resistencias subrepticias que muestran la capacidad de los internos de re-significar la psicología o rechazar, dentro de lo posible, la labor de los psicólogos. Cuestión que produce un juego dialéctico fundado desde la dicotomía rechazo/aprobación, y a la vez, un espacio de micro-resistencias ante el ejercicio del poder institucional desde los espacios psicológicos.

En otras palabras, se instaura un uso táctico de las situaciones que se presentan con los psicólogos. En ocasiones, este uso toma forma desde la dinámica castigo/recompensa, en la que se aprueba el rol cuando la interacción trae consigo ciertos privilegios. La labor del psicólogo es aprobada cuando la relación y la aceptación de los roles traen consigo recompensas hacia la condena, tal como la posibilidad de adelantar su salida mediante una adecuación (resultado de su presencia en terapia en la penal).

Tallerista R preguntó si todos iban a terapia ahí en la institución, Interno A dijo: "sí, si quieres salir más rápido" después preguntó: “¿alguno va a la terapia porque quiere o sólo 
porque les ayuda a salir más rápido?" la mayoría respondió que iban a terapia porque los ayudaba a salir antes (Bitácora 4).

Sea para reducir su condena ("sí, si quieres salir más rápido"), o para no realizar actividades impuestas por la institución; los espacios psicológicos les permiten resistir, es decir, re-significar el devenir penitenciario no desde el choque frontal o la resistencia abrupta, sino, desde el aprovechamiento táctico del contacto con los psicólogos y el discurso psicológico mediante acciones más indirectas. No obstante, esta cuestión no sólo produce una dialéctica poderresistencia, sino, además genera una concepción de la psicología desde dos lógicas dicotómicas: institucionalización-no institucionalización y anormal-locura.

\section{-Institucionalización del psicólogo}

La institucionalización del psicólogo es un elemento importante que participa en el modo que se significa la psicología por parte de las personas privadas de libertad. En términos concretos, este saber se define desde el rol que ostentan los psicólogos dentro de la institución (psicólogo/vigilante), lo cual, produce una dicotomía de aprobación/desaprobación. Por un lado, se encuentra la cara del rechazo, en la que el psicólogo se traza como un sujeto institucionalizado que se encarga indirectamente de vigilar, juzgar y castigar, y por otro, está la cara de aprobación cuando se les dibuja como canales de escucha, des-institucionalizados.

La desaprobación se produce cuando los internos relacionan al psicólogo como una autoridad encubierta, esto es, parte del personal que se encarga de vigilar y juzgar su condición. En tal sentido, acudir a la clínica se asocia a un montaje institucional, un momento de espionaje subrepticio. Desde aquí, el psicólogo actúa más como un vigilante, al grado en que el proceso terapéutico no se piensa como un proceso de escucha, sino como un espacio de extracción del yo que puede ser utilizado por el personal institucional ajeno al área psicológica (jueces, abogados, etc). El psicólogo, por ende, se le retrata como un agente encubierto que influye en las decisiones 
de la condena del interno: la adecuación, privilegios dentro de la institución, selección de módulo donde van a residir, etc.

T1 dijo: “¿y la psicología no la pondrían?” rápidamente interno $\mathrm{N}$ respondió con un tono de voz más alto y parecía algo molesto: "a esos no los vamos a poner, no hacen nada bien, para qué los ponemos, por ellos no me pude ir" T1 le preguntó qué había pasado, interno $\mathrm{N}$ respondió que le habían dado su adecuación y tenía la posibilidad de salir antes, pero que le hicieron muchas entrevistas en psicología y dijeron que era muy arrogante, por ese motivo no pudo salir antes... Interno $\mathrm{V}$, "es que puede ser que seas el mejor portado, como este wey (dijo señalando a interno N), pero con que el psicólogo diga algo, ya no te dejan salir (Bitácora 7).

En conclusión, la psicología se significa como un dispositivo de control. La frase "pero con que el psicólogo diga algo, ya no te dejan salir" no sólo muestra la desaprobación que genera, sino que vislumbra cómo los psicólogos, a partir de un saber legitimado, se piensan como agentes que facilitan el control, creando cuerpos dóciles, moldeables y clasificables. Por lo que desvalorizar como incompetentes a los psicólogos no tiene que ver con su capacidad de ejercer la psicología, sino con una respuesta ante la visible asociación con la institución y el control.

\section{-La psicología desde el binomio normal/anormal}

Dentro de las tensiones esbozadas de dominación y resistencias producidas por la misma lógica de la institución penitenciaria, la psicología (y la labor de los psicólogos) también se traza a partir del binomio anormal/normal. Un primer lado manifiesta una relación entre el psicólogo y el interno desde la noción de persona, esto es, en la medida de lo posible, desde la relación horizontal. De modo que la labor del psicólogo más que clasificar en términos patológicos o anormales, y de posicionarse desde un saber para patologizar a la población, se piensa desde la ayuda al interno. 
Mejor dicho, se significa al psicólogo desde la metáfora de la amistad, la cual, desdibuja los roles que se crean en la relación terapeuta-paciente.

TN les dijo, "bueno y si tuvieran que dejar la clínica pero la pueden cambiar como quieran, ¿como sería?". A respondió de la clínica, "sí la terapéutica, pero sin terapia, que sea ir a convivir ahí, a platicar" B siguió complementando a A, “...sí, y que ahí están ustedes las psicólogas, pero sin bata, de cotorreo, así normales, y sin ser psicólogas, si no como amigos pues, ¿como nosotros con ustedes no?" A se puso a dibujar la clínica sin terapia a un lado de la alberca (Bitácora 7).

[...] aquí es diferente con ustedes, sí nos escuchan y no nos tratan como locos (Bitácora 2).

Frases como "sí nos escuchan y no nos tratan como locos", y "pero sin bata, de cotorreo, así normales, y sin ser psicólogas, sino como amigos pues" no sólo muestran el deseo de los internos por establecer relaciones más horizontales, sino también visibilizan cómo la noción del psicólogo es reconocida cuando, paradójicamente, no se posiciona desde el saber psicológico junto con sus formas terapéuticas, ni determina las relaciones de dominación (bata) y dominado (sin bata), y sobre todo, cuando no los categoriza desde la anormalidad.

Int.1, sentado en la mesa al frente de TN, enderezó la espalda, se inclinó hacia delante y comenzó a hablar: "Sí, para los psicólogos nosotros somos los que según esto tenemos algo mal en la mente, problemas psicológicos; estamos locos. Sólo les importa lo que tienes en la mente, te la quieren arreglar (Bitácora 2).

[...] a mí tampoco me gusta la psicología. Te hacen sentir como que estás loco, como que tienes algo mal en tu cabeza, en tu mente (Bitácora 2).

Esta noción de rechazo se construye cuando el interno interpreta la psicología desde una lógica de alienación, o mejor dicho, desde un discurso que funciona como una doctrina que los traduce 
únicamente como sujetos anormales que deben ser tratados. En fin, no se trata de la necesidad de ser escuchados, sino que la relación psicólogo-interno no se oriente a la codificación del segundo como un ser distinto. "Sólo les importa lo que tienes en la mente, te la quieren arreglar", refleja el anhelo de los internos de ser tratados como algo más que objetos de estudio y el rechazo a la psicología por no tratarlos desde la paridad.

\section{Conclusiones}

Más que representar un espacio terapeútico (de escucha), la significación de la psicología por parte de los internos, adquiere sentido desde la tensión constante entre el ideal del psicólogo y el funcionamiento institucional. Esto implica que, generalmente, sea percibida como un dispositivo de poder (Foucault, 1975) y, sobre todo, que, encarnado por los psicólogos clínicos que trabajan en la institución, se configura como una autoridad moral que los vigila, y por otra parte, como un saber que se limita a buscar cualquier indicio personal para categorizarlo como loco, anormal, y por tanto, delincuente.

No obstante, sí hay situaciones en las que se desvincula la psicología del poder. En el momento en que esta no se evidencia como un dispositivo de poder (Foucault, 1975) adquiere una significación distinta, de aprobación. Específicamente, cuando la relación entre psicólogo e internos se instala, en la medida de lo posible, desde un espacio más horizontal (ergo: no vigilancia o espionaje), o cuando el lazo con el psicólogo se vive más desde la metáfora de la amistad (escucha y comprensión de situación). En suma, se traduce a un espacio terapéutico de ayuda, reflexión e introspección personal acompañada.

En otras palabras, tomando como referencia a García-Borés (1995), la psicología dentro del centro penitenciario vive en los dos pilares paradójicos que constituyen el funcionamiento de este tipo de lugares: 1) el régimen penitenciario y 2) el discurso de reinserción. La psicología, es, al mismo tiempo, espacio de control-castigo y momento de reflexión del yo. De ahí que cuando se habla a nivel abstracto, se le traza como una espacio de cambio y de ayuda, y a la vez, cuando se 
institucionaliza (se evidencia su lógica de poder) se le critica y rechaza por ser inculcada a la fuerza (Sanz, 2004), por mortificar a la persona desde la categoría anormal (Goffman, 1970), o simplemente por convertirse en un dispositivo de poder (Foucault, 1975) que utiliza la mancuerna jurídico-psicológico para cosificar al interno como un ser distinto/anormal y para vigilar, juzgar y controlar a las personas privadas de libertad en dicho centro.

Ahora bien, cada forma de significar tiene sus particularidades. La psicología desde el binomio normal/anormal se fundamenta más en el saber psicológico y la relación del psicólogo con los internos, que en las dinámicas penitenciarias de control. La desaprobación o aprobación de la psicología se centra más en la manera en que el psicólogo se desempeña en la terapia: la aprobación depende de una vinculación tenue dirigida más hacia la amistad y apoyo durante la condena; y el rechazo se produce si el lazo se orienta de forma directa hacia la exposición tajante de la intimidad del interno, al considerarlo más como una amenaza y un sujeto al cual examinar en el contexto de normalidad/anormalidad. Es decir, se le piensa como un aparato que construye al interno desde la diferencia y una plataforma de visibilización forzada de la intimidad del sujeto.

Por otro lado, la institucionalización de la psicología es un producto de la función del psicólogo dentro del aparato penitenciario, más que de la relación. De ahí la aprobación o desaprobación: entre más visible esta lógica es mayor el rechazo. En términos concretos, para los internos, lo psicólogos no sólo son representantes de un saber que pretende la reconstrucción y reflexión del sujeto, sino que también están anclados a un dispositivo de producción de verdad evidente. No sólo escuchan, sino también registran, examinan, develan, juzgan, en fin, traducen el comportamiento del interno y su intimidad a un lenguaje que permita tomar una decisión jurídica. Producen un diagnóstico que funciona como una verdad, y por ende, tiene repercusiones en la condena del interno. De ahí la desconfianza y desaprobación: más que personas, los psicólogos se perciben como un engrane más de la institución en su faceta de castigo, control y vigilancia.

Desde este punto de vista, la psicología vive una dicotomía aprobación/desaprobación que limita su campo de acción. Significación que no depende tanto de los métodos psicológicos o los 
procesos terapéuticos esbozados sino de dos elementos asociados explícitamente con la institución penitenciaria en su faceta material y simbólica: la relación producida por el objetivo de la terapia y la institucionalización del psicólogo. En fin, independientemente de su corriente, postura o forma de operar, ésta se significa como un instrumento de poder, lo cual, origina en automático su rechazo.

Hecho que debería re-dirigir la conversación académica y profesional acerca del papel los efectos de la psicología y los psicólogos dentro de las instituciones penitenciarias. Se trata de pasar de la discusión sobre ¿cuál es el mejor método? hacia ¿cuáles son las condiciones en las que operan los talleres e intervenciones psicológicas? Por más que se pretenda contribuir a la reinserción social de los internos desde programas psicológicos altamente especializados, el eje central del problema no está en los métodos empleados o posturas epistemológicas sobre cómo abordar la conducta delictiva; el problema está en el funcionamiento y las condiciones de las instituciones penales (una cuestión estructural, no de desempeño metodológico).

Habría que superar las preguntas canónica tales como ¿cuál es el mejor método psicológico en la cárcel? o ¿qué talleres producen mejores resultados? para encaminar la problemática a una dimensión más estructural: ¿cómo se puede construir un modelo psicológico que no se oriente a la búsqueda de la diferencia (anormalidad) y a la administración del yo?, ¿cómo deslindarse de la posición de poder que actualmente funge el psicólogo en las cárceles?, ¿cómo producir espacios de resingificación del yo que no se orienten, implícitamente, al diagnóstico jurídico? En definitiva, que la tarea intelectual ya no se enfoque únicamente en el método de la intervención psicológica, sino en las condiciones de poder que operan (y tienen un efecto) en dicho proceso.

\section{Referencias}

Baratta, A. (2004). Criminología crítica y crítica del derecho penal: Introducción a la sociología jurídica-penal. México DF: Siglo XIX 
Bergalli, R. (1976). ¿Readaptación social a través de la ejecución penal? Madrid: Instituto de Criminología. Universidad Complutense.

Certeau, M. D. (1980). La Invención de lo Cotidiano I Artes de hacer - 1ra reimpresión de la primera edición en español. México: Instituto tecnológico de estudios superiores de Occidente.

Clarke, V., \& Braun, V. (2006). Using thematic analysis in psychology. Qualitative research in Conde, P.-A. (2009). La creación y el espacio terapéutico en el medio penitenciario. Arteterapia, 4, 137-148.

Foucault, M. (1999). El orden del discurso. Barcelona, Tusquets.

Foucault, M. (1975). Vigilar y castigar: Nacimiento de la prisión. Buenos Aires: Siglo XXI Editores.

García-Borés, J. (2003). El impacto carcelario. En Sistema Penal y Problemas Sociales, 395-425.

García-Borés, J. M. (1995). La cárcel. En A. Aguirre y A. Rodríguez (Eds.), En Patios abiertos Patios Cerrados: Psicología cultural de las instituciones. (pp. 93-120). Barcelona: Marcombo.

Gergen, K. (1996). Realidades y relaciones. Aproximaciones a la construcción social. Barcelona: Paidós.

Gergen, K. (1997). Toward a cultural constructionist psychology. En Theory and Psychology 7: 31-36. Godenzzi, J.C. (1999) Pedagogía del encuentro. El sujeto, la convivencia y el conocimiento. Bulletin de l'Institut francais d'Études andines, 28(3), págs.. 323 - 328.

Goffman, E. (1970/2001). Internados: ensayos sobre la situación social de los enfermos mentales. Buenos Aires: Amorrortu.

González, L. (2002). La sistematización y el análisis de los datos cualitativos. Mejía R, Sandoval SA (Coords). Tras Las Vetas de La Investigación Cualitativa. Perspectivas y Acercamientos Desde La Práctica. Tlaquepaque: Instituto Tecnológico y de Estudios Superiores de Occidente, 155173.

Heleusa, F. (2006). Más allá de las rejas: en sus propias palabras. Revista Decisio. EDUC, MAYOAGOSTO. (p. 43- 47). 
Herrero, R. S. y León, J. L. (2006). Proceso de socialización y síntomas del estado de ánimo en delincuentes juveniles privados de libertad. Anuario de Psicología Clínica y de La Salud= Annuary of Clinical and Health Psychology, 2, 65-76.

Kouyoumdjian, L., \& Poblet, M. (2010). Un punto de fuga. La Educación En Cárceles, Aportes Desde EI Trabajo Social. Margen, 58(58), 1-7. Edición electrónica: http://www.margen.org/suscri/numero58.html. (8/12/10)

Pavarini, M. (1983). Control y dominación: teorías criminológicas burguesas y proyecto hegemónico. Siglo XXI.

Pavarini, M. (1987). La cárcel en Italia, entre la voluntad de descarcelación y necesidades disciplinarias. Poder y Control, 3. Psychology, 3(2), 77-1001. http://doi.org/http://dx.doi.org/10.1191/1478088706qp063oa

De Alós Moner, R.; Martín, A., Miguélez, F., y Gibert, F. (2009). ¿Sirve el trabajo penitenciario para la reinserción? Un estudio a partir de las opiniones de los presos de las cárceles de Cataluña. En Revista Española de Investigaciones Sociológicas (Reis), 127(1), 11-31.

Sanz, N. (2004). Alternativas a la prisión. Su viabilidad en las legislaciones centroamericana, española y mexicana. México DF: Instituto Nacional De Ciencias Penales. 